\title{
Seeing What We Expect to See in COVID-19
}

appreciate the effort among your staff that must be required to keep the articles in the American Journal of Neuroradiology current as we learn more about the potential for CNS involvement with coronavirus disease 2019 (COVID-19) infections.

The article in the September 2020 issue, "Anosmia in COVID19 Associated with Injury to the Olfactory Bulbs Evident on MRI," is certainly timely and of considerable interest because this symptom has been reported in many infected patients. ${ }^{1}$ In this article, the authors report their experience with the imaging of 5 patients whom they believe demonstrated abnormalities of the olfactory bulbs, though 1 of these 5 did not have anosmia. While their article appears to support the suspicion of many that COVID-19 directly involves the olfactory bulbs, I think we should be careful that we are not seeing only what we expect to see. In Fig 1, where the authors point to presumed hemorrhage (microbleeding) into the left olfactory bulb, there is also high signal in both inferior rectus muscles and bilateral frontal lobes just lateral to the olfactory sulcus on the precontrast scan and in the inferior frontal lobes on the postcontrast scan. Because it is most likely that these other areas of symmetric high signal along brain-bone-air interfaces in the orbits and frontal fossa are susceptibility artifacts, one could argue that the high signal that appears to be in the olfactory bulb is also artifactual. In support of that contention, the left and right olfactory bulbs do not appear enlarged or asymmetric on the STIR scan, surprising with presumed hemorrhage.

It is entirely possible, however, that one or more of the other cases in which only postcontrast imaging is provided have real enhancement of the olfactory bulbs. However, they do not appear to be enlarged, and this feature differs from a report in Neurology, in which transient enlargement without particularly evident signal change was illustrated in a patient with COVID-19 and anosmia, ${ }^{2}$ albeit measured on MR imaging that appears to be acquired on different scanners or at least with different techniques.

This discrepancy among early reports supports the commentary in the same issue, "Level of Evidence during COVID-19 Pandemic: Making the Case for Case Series and Case Reports." ${ }^{3}$ I agree that rapid publication of case reports of imaging findings with COVID-19 will, in time, benefit our patients, but in the meantime, we need to be particularly critical about the quality of these early reports and avoid reaching any conclusions until there is consistent and reliable evidence regarding CNS involvement from COVID-19 infection.

\section{REFERENCES}

1. Aragão MF, Leal MC, Filho OQ, et al. Anosmia in COVID-19 associated with injury to the olfactory bulbs evident on MRI. AJNR Am J Neuroradiol 2020;41:1703-06 CrossRef Medline

2. Laurendon T, Radulesco T, Mugnier J, et al. Bilateral transient olfactory bulb edema during COVID-19-related anosmia. Neurology 2020;95:224-25 CrossRef Medline

3. Ikuta I. Level of evidence during COVID-19 pandemic: making the case for case series and case reports. AJNR Am J Neuroradiol 2020;41;1646 CrossRef Medline
(D) Aamourian

Department of Radiology Penn State Health Milton S. Hershey Medical Center, Penn State College of Medicine Hershey, Pennsylvania 